\title{
Coordination in urban water supply networks using distributed model predictive control
}

\author{
S. Leirens, C. Zamora, R.R. Negenborn, and B. De Schutter
}

\begin{abstract}
Urban water supply networks are large-scale systems that transport potable water over vast geographical areas to millions of consumers. A safe and efficient operation of these networks is crucial, as without it living in today's cities would be impossible. To achieve an adequate operation, these networks are equipped with actuators like pumps and valves, which are used to maintain water pressures and flows within safe margins. Currently, these actuators are controlled in a decentralized way using local controllers that only use local information and that do not take into account the presence of other controllers. As a result, water supply networks regularly experience pressure drops and interruptions of water supply when there is an unexpected increase in water demand. To improve performance the actions of the local controllers should be coordinated. Implementing a centralized control scheme is not tractable due to the large-scale nature of these networks. Therefore, this paper proposes the application of a distributed control scheme for control of urban water supply networks. The scheme is based on local model predictive control (MPC) strategies and a parallel coordination scheme that implements cooperation among the local MPC controllers. A simulation study based on a part of the urban water supply network of Bogotá, the capital of Colombia, illustrates the potential of the approach.
\end{abstract}

\section{INTRODUCTION}

\section{A. Urban water supply networks}

Urban water supply networks form the link between drinking water supply and drinking water consumers. These large-scale networks are vital for the survival of urban life, for maintaining a healthy level of economic development, and for the continuous operation of factories and hospitals [1]. Water supply networks consist of so-called transmission mains, distribution mains, and service lines. Transmission mains convey large amounts of water over large distances; distribution mains provide intermediate steps toward delivering water to the end customers; and service lines transmit the water from the distribution mains to the end customers. The transmission and distribution network can have a topology that is looped, branched, or a combination of these two [2].

In water supply networks pumps and valves are the control elements that enable the delivery of water to the consumers taking into account water flow and pressure constraints. The most frequently used type of pump in water supply networks is the centrifugal pump, which consists of a motor and an impeller. In this device, the mechanical energy of the rotating

S. Leirens and C. Zamora are with the Departamento de Ingeniería Eléctrica y Electrónica, Universidad de Los Andes, Bogotá, Colombia, \{sleirens, cp.zamora 71$\}$ @uniandes.edu.co. R.R. Negenborn and B. De Schutter are with the Delft Center for Systems and Control, Delft University of Technology, Delft, The Netherlands, r.r.negenbornatudelft.nl, b. deschutteratudelft.nl. impeller is injected into the water, resulting in an increase in pressure [3]. Valves are actuators that vary resistance to change the water flow and that can be operated manually or automatically. Valves can be classified as pressure reducing valves, pressure sustaining valves, and flow control valves. Pressure reducing valves throttle automatically to prevent the downstream hydraulic grade from exceeding a set-point. Pressure sustaining valves throttle the flow automatically to prevent the upstream hydraulic grade from dropping below a set-point. Flow control valves throttle automatically to limit the flow rate passing through the valve to a maximum value [2]. In the system under study in this paper each of these types of valves is present.

\section{B. Control of water supply networks}

In water supply networks the primary control objective is to guarantee the delivery of the right amount of water at the right place at the right time. Hereby, pressure at particular locations in the network and flows through particular pipes of the network have to stay within acceptable limits. A low flow rate can lead to water quality problems due to the growth of undesirable microorganisms and the accumulation of metals and salts on the wall of the transmission and distribution pipes. Too large pressure values increase water losses (due to pipe waste) and incur a larger blow-out probability [2].

The control system of a water supply network has to determine the settings for the actuators in the network in such a way that the control objectives are achieved. Hereby, constraints on the range of possible control signals have to be respected, even when there are unexpected changes in water demand. Model-based predictive control seems ideally suited for this as detailed below.

\section{Model predictive control}

Model predictive control (MPC) is a control approach in which at discrete time steps actions are determined by solving an optimization problem, taking into account the control objectives, predicted dynamics of the network over a certain prediction horizon, and operational constraints [4]. MPC has been widely used in the process industry and is now gaining increasing attention in other fields, including control of water networks [5]-[8]. The main advantages of using MPC are that multiple-input multiple-output systems can be handled in an integrated way, and that constraints, long time delays, and known information (e.g., regarding future disturbances) can be taken into account in a straightforward fashion. Each of these advantages can also be taken benefit from in the domain of water supply networks. 
Due to the complexity and the large size of water networks, control of such systems in general, however, cannot be done in a centralized way in which a single controller operates the full network from a single location. Instead of collecting measurements from the whole system and determining actions from a single location, control is typically spread over several local control agencies, each controlling a particular part of the network. Since the 90s various distributed MPC applications have been investigated for water networks. E.g., in [9], [10] a decentralized adaptive control approach for a 40-kilometer long canal in France is considered; in [11] a decentralized unconstrained predictive controller for water delivery canals is presented; and in [12] distributed MPC for irrigation canals is considered.

In this paper we propose to employ distributed MPC for control of large-scale urban water supply networks, in particular for the water supply network of Bogotá, Colombia.

\section{Outline}

This paper is organized as follows. In Section II models for the components of water supply networks are given and the water supply network of Bogotá, which we use as a system for case studies, is presented. In Section III the details of the distributed MPC scheme proposed for control of water supply networks are given. Section IV discusses a simulation experiment in which the distributed MPC scheme is applied for control of a part of the water supply network of Bogotá. Section V concludes this paper.

\section{WATER SUPPLY NETWORK MODELING}

A water supply system consists of a large number of interconnected hydraulic elements, which can be classified as either being active or passive elements [13]. Active elements (e.g., pumps and valves) can alter the flow rate of water in specific parts of the system. Passive elements (e.g., pipes, tanks, and reservoirs) do not directly alter the flow rates. The behavior of these elements is formalized below.

\section{A. Pumps}

Pumps add mechanical energy transformed into additional head to the hydraulic system [2], [13]. The head $h_{\mathrm{p}}[\mathrm{m}]$ varies nonlinearly with the speed $N[\mathrm{rpm}]$ and the flow $Q_{\mathrm{p}}\left[\mathrm{m}^{3} / \mathrm{s}\right]$. The head $h_{\mathrm{p}}$ of $n$ variable-speed pumps running in parallel is at time $t$ given by:

$$
h_{\mathrm{p}}(t)=A_{0} N(t)^{2}+\frac{B_{0}}{n} N(t) Q_{\mathrm{p}}(t)-\frac{C_{0}}{n^{2}} Q_{\mathrm{p}}^{2}(t),
$$

where $A_{0}, B_{0}, C_{0}$ are suitable constants for a particular pump, as provided by the manufacturer.

\section{B. Pipes}

Pipes convey flow as water moves from one junction to another. Let a pipe section have length $l_{\mathrm{p}}[\mathrm{m}]$, cross sectional area $A_{\mathrm{p}}\left[\mathrm{m}^{2}\right]$, and head difference between the two ends of the pipe $\Delta h_{\mathrm{p}}[\mathrm{m}]$. The evolution of the flow $Q_{\mathrm{p}}(t)\left[\mathrm{m}^{3} / \mathrm{s}\right]$ through the pipe is given by:

$$
\frac{\mathrm{d} Q_{\mathrm{p}}(t)}{\mathrm{d} t}=\frac{g A_{\mathrm{p}}}{l_{\mathrm{p}}}\left(\Delta h_{\mathrm{p}}(t)-h_{\mathrm{loss}}(t)\right)
$$

where $h_{\text {loss }}$ denotes the total head loss along the piping section and $g$ is the gravitational acceleration. The total head loss is given by [13]:

$$
h_{\mathrm{loss}}(t)=h_{\mathrm{loss}, f_{\mathrm{p}}}(t)+h_{\mathrm{loss}, \mathrm{m}}(t),
$$

where $h_{\mathrm{loss}, f_{\mathrm{p}}}$ represents friction losses and $h_{\mathrm{loss}, \mathrm{m}}$ represents minor local losses. The friction losses are computed using Darcy-Weisbach's friction model [3]:

$$
h_{\mathrm{loss}, f_{\mathrm{p}}}(t)=\left(\frac{c_{f_{\mathrm{p}}} l_{\mathrm{p}}}{D_{\mathrm{p}} A_{\mathrm{p}}^{2} 2 g}\right) Q_{\mathrm{p}}^{2}(t),
$$

where $D_{\mathrm{p}}$ is the inner diameter of the pipe [m], and $c_{f_{\mathrm{p}}}$ is a roughness coefficient. This friction coefficient varies depending on the surface roughness of the pipe. Colebrook and White [3] suggest to use the following empirical equation to determine this friction coefficient:

$$
\frac{1}{\sqrt{c_{f_{\mathrm{p}}}}}=-4 \log _{10}\left(\frac{\epsilon / D_{\mathrm{p}}}{3.71}+\frac{2.51}{2 \sqrt{2 c_{f_{\mathrm{p}}}} N_{\mathrm{R}}}\right),
$$

where $\epsilon[\mathrm{m}]$ is the roughness of the pipe and $N_{\mathrm{R}}$ is its Reynolds number. This equation can be solved using standard numerical methods to find roots of nonlinear algebraic equations, such as bisection or Newton-Raphson methods [13].

The minor local losses $h_{\text {loss,m }}[\mathrm{m}]$ are caused by contractions, fittings, expansions, bends, valves, and changes in flow in the pipeline. Minor head losses are expressed as a function of the water velocity in the pipe and depend on $K_{\mathrm{m}}$, the head loss coefficient, as follows:

$$
h_{\mathrm{loss}, \mathrm{m}}(t)=\left(\frac{K_{\mathrm{m}}}{A_{\mathrm{p}}^{2} 2 g}\right) Q_{\mathrm{p}}^{2}(t) .
$$

\section{Nodes}

The following conservation of mass principle is considered at nodes:

$$
Q_{i}(t)-C_{i}(t)-\frac{\mathrm{d} S_{i}(t)}{\mathrm{d} t}=0
$$

where $Q_{i}$ is the total inflow into node $i\left[\mathrm{~m}^{3} / \mathrm{s}\right], C_{i}$ is the water used at node $i\left[\mathrm{~m}^{3} / \mathrm{s}\right]$, and $\frac{\mathrm{d} S_{i}}{\mathrm{~d} t}$ represents change in storage $\left[\mathrm{m}^{3} / \mathrm{s}\right]$. The conservation of mass equation is applied at all junction nodes and tanks in a network [14].

\section{Water supply network of Bogotá}

Bogotá, the capital of Colombia, is located on a high plateau mountain in the Andes Region, elevated $2630 \mathrm{~m}$ above sea level. Bogotá has a population of over 7 million inhabitants, who have to be supplied with water via Bogota's water supply network, see Figure 1. This water supply network receives water from four treatment plants: Wiesner, El Dorado, Tibitoc, and Tibitoc Alto. In each of these plants the water undergoes several processing steps in order to ensure water that is safe for human consumption. After these processing steps, the water is distributed via the transmission and distribution mains of the supply network to the metropolitan area. The water supply network of 
Bogotá includes 33 pumping stations, 55 storage tanks, and approximatively $500 \mathrm{~km}$ of 12 inch or larger pipes ${ }^{1}$.

In this paper we consider control of a particular region of the complete network (as indicated in Figure 1) as a first step towards control of the complete water supply network. To obtain a dynamic model for this system, parameters of pipes (diameters, nodes, lengths, and roughness), junctions (elevation, demands, and consumption patterns), as well as topographic altitudes of reservoirs, valves, and pumps have been collected. This information has then been used to develop an overall state space representation of the following form:

$$
\dot{\mathbf{x}}(t)=f(\mathbf{x}(t), \mathbf{u}(t), \mathbf{d}(t)),
$$

where $\mathbf{x}$ is the state (the flow through pipes and the head in nodes), $\mathbf{u}$ is the control input (loss coefficient of valves and pressure injection of pumps), $\mathbf{d}$ is the exogenous disturbance input (the consumption pattern), and $f$ is the nonlinear state transition function.

The actuators in the network should be set in such a way that the control objectives are achieved. Due to the large scale of the network, centralized control is not tractable. Below we present how distributed MPC can be used to coordinate the actions taken locally throughout the network instead.

\section{DISTRIBUTED MODEL PREDICTIVE CONTROL}

Using distributed MPC, the control of the system (8) is divided over multiple controllers. An individual controller on the one hand obtains measurements from and determines actions for its part of the network, and on the other hand communicates with other controllers in order to obtain coordination and to improve the overall network performance. To determine which actions to take, each controller uses an MPC strategy.

\section{A. Model predictive control}

In this paper, it is assumed that the dynamics of the prediction model of subnetwork $i$ are represented by a timeinvariant linear (or linearized) model described in a discretetime state-space framework:

$$
\begin{aligned}
& \mathbf{x}_{i}(k+1)=\mathbf{A}_{i} \mathbf{x}_{i}(k)+\mathbf{B}_{1, i} \mathbf{u}_{i}(k)+\mathbf{B}_{2, i} \mathbf{d}_{i}(k)+\mathbf{B}_{3, i} \mathbf{v}_{i}(k) \\
& \mathbf{y}_{i}(k)=\mathbf{C}_{i} \mathbf{x}_{i}(k)+\mathbf{D}_{1, i} \mathbf{u}_{i}(k)+\mathbf{D}_{2, i} \mathbf{d}_{i}(k)+\mathbf{D}_{3, i} \mathbf{v}_{i}(k),
\end{aligned}
$$

where $\mathbf{x}_{i} \in \mathbb{R}^{n_{\mathbf{x}_{i}}}$ is the state, $\mathbf{u}_{i} \in \mathbb{R}^{n_{\mathbf{u}_{i}}}$ is the control input, $\mathbf{y}_{i} \in \mathbb{R}^{n_{\mathbf{y}_{i}}}$ is the output, $\mathbf{d}_{i} \in \mathbb{R}^{n_{\mathbf{d}_{i}}}$ is the exogenous disturbance input (assumed to measured or estimated), and $\mathbf{v}_{i} \in \mathbb{R}^{n_{\mathbf{v}_{i}}}$ represents the influence of the neighboring subnetworks on subnetwork $i$. In the above equations $k \in \mathbb{N}$ is the (discrete) time instant, where discrete time $k$ corresponds to continuous time $k T$, where $T$ is the sample time of the discrete-time model (9). From a control point of view, note that input $\mathbf{v}_{i}$ has to be obtained via communication between local controllers, leading to a distributed control scheme, or can be simply ignored or assumed constant, leading to decentralized control. Obviously, if the network is strongly interconnected,

\footnotetext{
${ }^{1}$ http: //web. acueducto.com.co/.
}

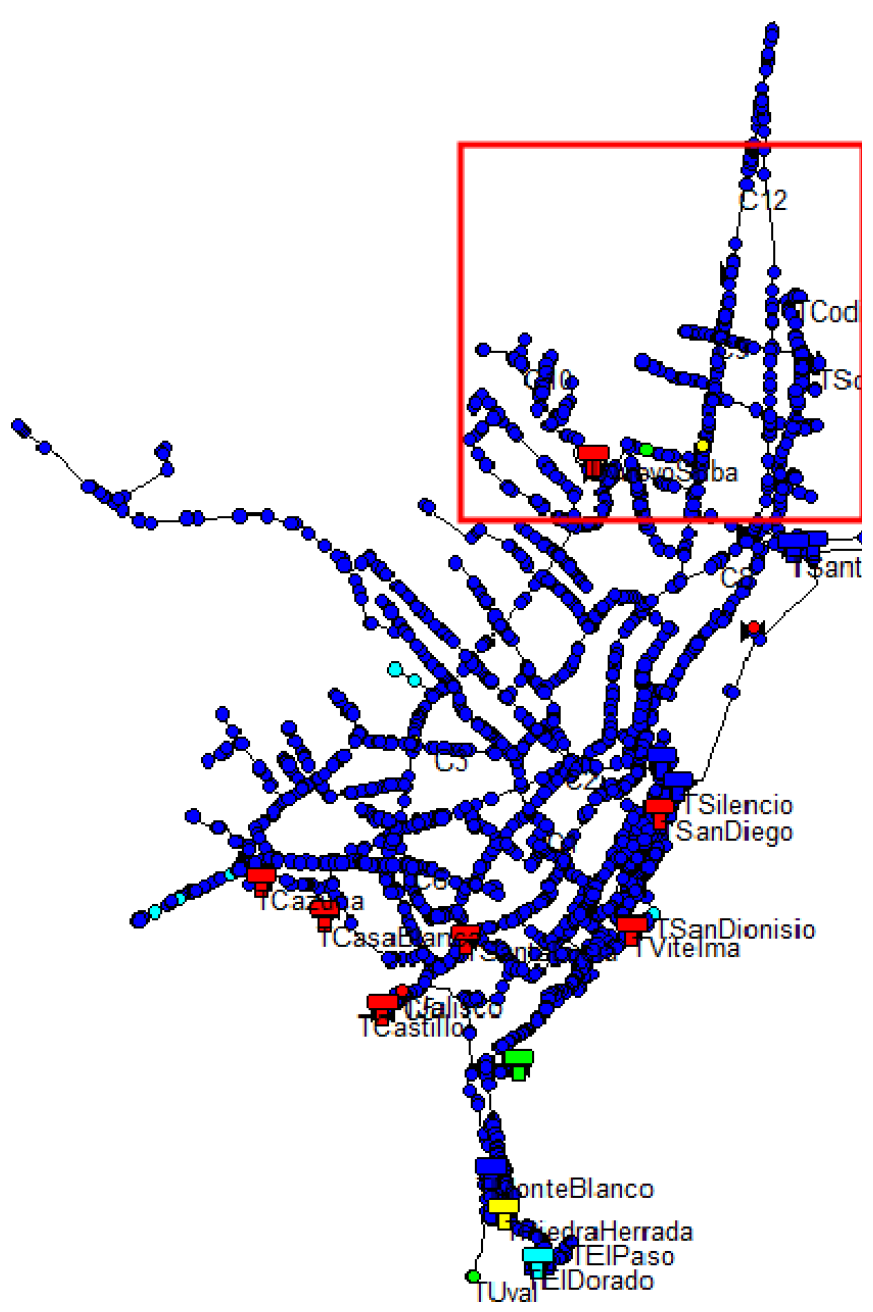

Fig. 1. Water supply network of Bogotá and the selected part for application of distributed control (inside the rectangle). Blue circles are consumption nodes, red and yellow symbols are storage tanks, cyan symbols are reservoirs, black lines are pipes.

the influence of input $\mathbf{v}_{i}$ cannot be neglected without a significant decrease in performance and robustness of the control system.

Consider that subnetwork $i$ has to be operated under (linear) constraints on input, state and output:

$$
\mathbf{E}_{i} \mathbf{x}_{i}(k)+\mathbf{F}_{i} \mathbf{u}_{i}(k)+\mathbf{G}_{i} \mathbf{y}_{i}(k)+\mathbf{h}_{i} \leq 0, \quad \forall k
$$

where $\mathbf{E}_{i}, \mathbf{F}_{i}, \mathbf{G}_{i}$, and $\mathbf{h}_{i}$ are assumed to be constant.

For the completeness of the paper, the principles of MPC are now briefly reviewed [4]. MPC is a model-based control method in which a model of the system to control is used to predict its behavior over a finite time horizon in the future. In a state-space framework, the following steps are considered: (1) at current control sample instant, the state of the system is measured (or estimated); (2) an optimization routine is used to find an optimal sequence of control inputs in the future that minimize an objective function subject to constraints; (3) the first control input from the optimal sequence is applied to the system, and steps (1)-(3) are repeated at the next control sample instant, and so on. The fact that a new measurement 
of the state is used at each time instant gives a feedback policy.

$$
\text { Let } \tilde{\mathbf{x}}_{i}(k+1)=\left[\begin{array}{lll}
\mathbf{x}_{i}^{\mathrm{T}}(k+1) & \cdots & \mathbf{x}_{i}^{\mathrm{T}}(k+N)
\end{array}\right]^{\mathrm{T}}, \tilde{\mathbf{u}}_{i}(k)=\left[\begin{array}{ll}
\mathbf{u}_{i}^{\mathrm{T}}(k) &
\end{array}\right.
$$
$\left.\begin{array}{lll}\cdots & \mathbf{u}_{i}^{\mathrm{T}}(k+N-1)\end{array}\right]^{\mathrm{T}}$ and $\tilde{\mathbf{y}}_{i}(k)=\left[\begin{array}{llll}\mathbf{y}_{i}^{\mathrm{T}}(k) & \cdots & \mathbf{y}_{i}^{\mathrm{T}}(k+N-1)\end{array}\right]^{\mathrm{T}}$ be the predicted state, input, and output sequences respectively. Here, the superscript T denotes transpose and $N$ is the length of the prediction horizon. In this paper, the prediction horizon and the control horizon have the same length $N$.

The following notations are used in the following: $\|\mathbf{z}\|_{Q}^{2} \triangleq$ $\mathbf{z}^{\mathrm{T}} \mathbf{Q z} ; \Delta \mathbf{u}(k) \triangleq \mathbf{u}(k)-\mathbf{u}(k-1) ; \operatorname{and} \operatorname{diag}\left(\mathbf{P}_{1}, \ldots, \mathbf{P}_{N}\right)$ is a block diagonal matrix of which the diagonal blocks are $\mathbf{P}_{i}(i=$ $1, \ldots, N)$. For subsystem $i$, consider the following objective function:

$$
\begin{aligned}
J_{\text {local }, i} & \left(\tilde{\mathbf{x}}_{i}(k+1), \tilde{\mathbf{u}}_{i}(k), \tilde{\mathbf{y}}_{i}(k)\right) \\
= & \left\|\tilde{\mathbf{x}}_{i}(k+1)-\tilde{\mathbf{x}}_{i, \text { ref }}\right\|_{\mathbf{Q}_{\tilde{\mathbf{x}}_{i}}}^{2}+\left\|\Delta \tilde{\mathbf{u}}_{i}(k)\right\|_{\mathbf{Q}_{\Delta \tilde{\mathbf{u}}_{i}}}^{2} \\
& +\left\|\tilde{\mathbf{u}}_{i}(k)-\tilde{\mathbf{u}}_{i, \mathrm{ref}}\right\|_{\mathbf{Q}_{\tilde{\mathbf{u}}_{i}}}^{2}+\left\|\tilde{\mathbf{y}}_{i}(k)-\tilde{\mathbf{y}}_{i, \text { ref }}\right\|_{\mathbf{Q}_{\tilde{\mathbf{y}}_{i}}}^{2},
\end{aligned}
$$

where $\mathbf{Q}_{\tilde{\mathbf{x}}_{i}}=\operatorname{diag}\left(\mathbf{Q}_{\mathbf{x}_{i}}, \ldots, \quad \mathbf{Q}_{\mathbf{x}_{i}}\right)$ is positive definite, and the sequences of the sequence of set-points $\tilde{\mathbf{x}}_{i, \text { ref }}=$ $\left[\mathbf{x}_{i, \text { ref }}^{\mathrm{T}} \ldots \mathbf{x}_{i, \text { ref }}^{\mathrm{T}}\right]^{\mathrm{T}}$, are considered constant during the prediction. The same holds for $\mathbf{Q}_{\tilde{\mathbf{u}}_{i}}$ and $\mathbf{Q}_{\tilde{\mathbf{y}}_{i}}$, and $\tilde{\mathbf{u}}_{i, \text { ref }}$ and $\tilde{\mathbf{y}}_{i, \text { ref. }}$.

Now, consider the local optimization problem $\mathcal{P}_{\text {local }, i}$ :

$$
\min _{\tilde{\mathbf{x}}_{i}(k+1), \tilde{\mathbf{u}}_{i}(k), \tilde{\mathbf{y}}_{i}(k)} J_{\text {local }, i}\left(\tilde{\mathbf{x}}_{i}(k+1), \tilde{\mathbf{u}}_{i}(k), \tilde{\mathbf{y}}_{i}(k)\right)
$$

subject to the dynamics (9) of subnetwork $i$, local constraints (10) on $\mathbf{x}_{i}(k), \mathbf{u}_{i}(k)$, and $\mathbf{y}_{i}(k)$, and let us for now ignore the input $\mathbf{v}_{i}(k+j)$, for $j=0, \ldots, N-1$. Define $\tilde{\mathbf{V}}_{\text {local }, i}(k)=$ $\left[\tilde{\mathbf{x}}_{i}(k+1)^{\mathrm{T}} \tilde{\mathbf{u}}_{i}(k)^{\mathrm{T}} \tilde{\mathbf{y}}_{i}(k)^{\mathrm{T}}\right]^{\mathrm{T}}$. Then,

$$
\begin{aligned}
& J_{\text {local }, i}\left(\tilde{\mathbf{V}}_{\text {local }, i}(k)\right) \\
& \quad=\tilde{\mathbf{V}}_{\text {local }, i}^{\mathrm{T}}(k) \mathbf{Q}_{\text {local }, i} \tilde{\mathbf{V}}_{\text {local }, i}(k)+\mathbf{f}_{\text {local }, i}^{\mathrm{T}} \tilde{\mathbf{V}}_{\text {local }, i}(k)+c_{\text {local }, i}
\end{aligned}
$$

with $\mathbf{Q}_{\text {local }, i}=\operatorname{diag}\left(\mathbf{Q}_{\tilde{\mathbf{x}}_{i}}, \mathbf{Q}_{\tilde{\mathbf{u}}_{i}}^{\prime}, \mathbf{Q}_{\tilde{\mathbf{y}}_{i}}\right), \mathbf{f}_{\text {local }, i}=\left[\mathbf{f}_{\tilde{\mathbf{x}}_{i}}^{\mathrm{T}} \mathbf{f}_{\tilde{\mathbf{u}}_{i}}^{\prime \mathrm{T}} \mathbf{f}_{\tilde{\mathbf{y}}_{i}}^{\mathrm{T}}\right]^{\mathrm{T}}, c_{\text {local }, i}$ a constant, and where $\mathbf{Q}_{\tilde{\mathbf{u}}_{i}}^{\prime}$ and $\mathbf{f}_{\tilde{\mathbf{u}}_{i}}^{\prime}$ account for both terms in $\tilde{\mathbf{u}}_{i}$ and $\Delta \tilde{\mathbf{u}}_{i}$ in cost function (11). Similarly, the constraints in $\mathcal{P}_{\text {local }, i}$ can be formulated as follows:

$$
\begin{aligned}
\mathbf{A}_{\text {in }, \text { local }, i} \tilde{\mathbf{V}}_{\text {local }}(k) & \leq \mathbf{b}_{\text {in }, \text { local }, i} \\
\mathbf{A}_{\text {eq }, \text { local }, i} \tilde{\mathbf{V}}_{\text {local }}(k) & =\mathbf{b}_{\text {eq }, \text { local }, i}
\end{aligned}
$$

$\mathcal{P}_{\text {local }, i}$ has now been reformulated as a standard quadratic programming $(\mathrm{QP})$ problem.

Up to now, the influence of the neighbors of subnetwork $i$ $\left(\mathbf{v}_{i}\right)$ has been ignored. As explained before, this influence has to be taken into account, and so communication between controllers is required at each time instant. For this purpose, the distributed control scheme based on local MPC controllers as introduced in [15] is described next.

\section{B. Distributed control scheme}

Let $\mathcal{N}_{i}=\left\{j_{i, 1}, \cdots, j_{i, m_{i}}\right\}$ be the set of indices of neighbors of subnetwork $i$, i.e., the subnetworks by which the dynamics of subnetwork $i$ are influenced. Let $\mathbf{w}_{\text {in }, j i}$ and $\mathbf{w}_{\text {out }, j i}$ be the so-called interconnecting input and output variables that subnetwork $i$ shares with a neighboring subnetwork $j$. Actually, interconnecting constraints

$$
\begin{aligned}
\mathbf{w}_{\text {in }, j i}(k) & =\mathbf{w}_{\text {out }, i j}(k) \\
\mathbf{w}_{\text {out }, j i}(k) & =\mathbf{w}_{\text {in }, i j}(k)
\end{aligned}
$$

should be added to the local control problem of controller $i, \forall j \in \mathcal{N}_{i}$ and for $k=0, \ldots, N-1$. However, adding such constraints leads to a nonseparable local optimization problem, since it cannot be solved using only local variables. To deal with constraints (16), a separable augmented Lagrange formulation is used [15]. Constraints (16) are added to the local objective function of controller $i$ in the form of linear terms in the Lagrange multipliers and quadratic terms in the interconnecting variables [15].

Then each local MPC controller computes optimal sequences as before, but now taking also the interconnecting variables in the sequences of variables to be optimized and now using fixed values for the Lagrange multipliers. This way, each local controller is able to communicate to its neighbors the optimal values, according to its local viewpoint, of the interconnecting variables. Convergence is obtained by updating the Lagrange multipliers using the solution of the optimizations and then repeating the optimizations until the Lagrange multipliers do not change anymore (with respect to small numerical tolerance) from one iteration to the next. Under convexity assumptions on the objective function and linearity of the subnetwork model constraints, solving the distributed control problem in such a way leads to the optimal solution of the centralized control problem that considers the entire network.

The coordination process is realized using a parallel coordination scheme [15] in which at time $k$ the following steps are performed: (1) all controllers compute their optimal sequences in parallel, (2) controllers communicate their optimal sequences of interconnecting input and output variables to their neighbors, (3) controllers update their Lagrange multipliers and communicate them to their neighbors. Steps (1)(4) are repeated until convergence (agreement) is reached.

For controller $i$, let the Lagrange multipliers $\boldsymbol{\lambda}_{\text {in, }, j i}(k)$ be associated to $\mathbf{w}_{\text {in }, j i}(k)$. As before, a tilde over a variable will denote a sequence over the prediction horizon $N$, so let $\tilde{\boldsymbol{\lambda}}_{\mathrm{in}, \mathcal{N}_{i}}(k)=\left[\begin{array}{llll}\tilde{\boldsymbol{\lambda}}_{\mathrm{in}, i j_{i, 1}}^{\mathrm{T}}(k) & \cdots & \tilde{\boldsymbol{\lambda}}_{\mathrm{in}, i j_{i, m_{i}}}^{\mathrm{T}}(k)\end{array}\right]^{\mathrm{T}}$ be the Lagrange multipliers that will be received by controller $i$ from its $m_{i}$ neighboring controllers. Let $\tilde{\mathbf{w}}_{\mathrm{in}, i}(k)=\left[\begin{array}{lll}\tilde{\mathbf{w}}_{\mathrm{in}, j_{i, 1} i}^{\mathrm{T}}(k) & \cdots & \tilde{\mathbf{w}}_{\mathrm{in}, j_{i, m_{i} i}}^{\mathrm{T}}(k)\end{array}\right]^{\mathrm{T}}$ be the interconnecting input variables of controller $i$ and let $\tilde{\mathbf{w}}_{\text {out }, \mathcal{N}_{i}}(k)=\left[\begin{array}{llll}\tilde{\mathbf{w}}_{\text {out }, i j_{i, 1}}^{\mathrm{T}}(k) & \cdots & \tilde{\mathbf{w}}_{\text {out }, i j_{i, m_{i}}}^{\mathrm{T}}(k)\end{array}\right]^{\mathrm{T}}$ be the interconnecting output variables of the $m_{i}$ neighboring subsystems that are interconnecting input variables of subsystem $i$. Let $\tilde{\mathbf{w}}_{\text {out }, i}(k)$ and $\tilde{\mathbf{w}}_{\text {in, } \mathcal{N}_{i}}(k)$ be defined in the same way. Then we have

$$
\begin{aligned}
\tilde{\mathbf{v}}_{i}(k) & =\mathbf{P}_{i} \tilde{\mathbf{w}}_{\text {in }, i}(k) \\
\tilde{\mathbf{w}}_{\text {out }, i}(k) & =\mathbf{K}_{i}\left[\begin{array}{ll}
\tilde{\mathbf{x}}_{i}(k)^{\mathrm{T}} & \tilde{\mathbf{u}}_{i}(k)^{\mathrm{T}} \tilde{\mathbf{y}}_{i}(k)^{\mathrm{T}}
\end{array}\right]^{\mathrm{T}},
\end{aligned}
$$


where $\mathbf{P}_{i}$ and $\mathbf{K}_{i}$ are matrices that contain zeros everywhere, except for a single 1 per row.

Consider the augmented local optimization problem for controller $i$ that has to be solved at each iteration of the coordination process:

$$
\begin{aligned}
& \min _{\substack{\tilde{\mathbf{x}}_{i}(k), \tilde{\mathbf{u}}_{i}(k), \tilde{\mathbf{y}}_{i}(k), \tilde{\mathbf{w}}_{\text {in }, i}(k), \tilde{\mathbf{w}}_{\text {out }, i}(k)}} J_{\text {local }, i}\left(\tilde{\mathbf{x}}_{i}(k), \tilde{\mathbf{u}}_{i}(k), \tilde{\mathbf{y}}_{i}(k)\right) \\
& +J_{\text {inter }, i}\left(\tilde{\mathbf{w}}_{\text {in }, i}(k), \tilde{\mathbf{w}}_{\text {out }, i}(k)\right)
\end{aligned}
$$

subject to the dynamics (9) of subnetwork $i$ and local constraints (10) on $\mathbf{x}_{i}(k), \mathbf{u}_{i}(k)$, and $\mathbf{y}_{i}(k)$, and equalities (18)-(19), for $k=0, \ldots, N-1$. This augmented formulation includes a new term $J_{\text {inter }, i}$ that is defined as follows:

$$
\begin{aligned}
& J_{\text {inter }, i}\left(\tilde{\mathbf{w}}_{\text {in }, i}(k), \tilde{\mathbf{w}}_{\text {out }, i}(k)\right) \\
& =\tilde{\lambda}_{\text {in }, i}^{\mathrm{T}}(k) \tilde{\mathbf{w}}_{\text {in }, i}(k)-\tilde{\boldsymbol{\lambda}}_{\text {in }, \mathcal{N}_{i}}^{\mathrm{T}}(k) \tilde{\mathbf{w}}_{\text {out }, i}(k) \\
& \quad+\frac{\gamma_{\mathrm{c}}}{2}\left(\left\|\tilde{\mathbf{w}}_{\text {in }, i}(k)-\tilde{\mathbf{w}}_{\text {out }, \mathcal{N}_{i}}(k-1)\right\|^{2}\right. \\
& \left.\quad+\left\|\tilde{\mathbf{w}}_{\text {out }, i}(k)-\tilde{\mathbf{w}}_{\text {in }, \mathcal{N}_{i}}(k-1)\right\|^{2}\right),
\end{aligned}
$$

where the linear terms involve the Lagrange multipliers $\tilde{\boldsymbol{\lambda}}_{\mathrm{in}, i}(k)$ and $\tilde{\boldsymbol{\lambda}}_{\mathrm{in}, \mathcal{N}_{i}}(k)$, and the quadratic terms with tuning parameter $\gamma_{\mathrm{c}}$ penalize the deviation of $\tilde{\mathbf{w}}_{\mathrm{in}, i}(k)$ and $\tilde{\mathbf{w}}_{\text {out }, i}(k)$ from the interconnecting variables iterates that were computed at the last iteration: $\tilde{\mathbf{w}}_{\text {out }, \mathcal{N}_{i}}(k-1)$ and $\tilde{\mathbf{w}}_{\text {in }, \mathcal{N}_{i}}(k-1)$.

The Lagrange multipliers of controller $i$ are updated, at iteration $s$, as follows:

$$
\tilde{\boldsymbol{\lambda}}_{\mathrm{in}, i}^{(s+1)}(k)=\tilde{\boldsymbol{\lambda}}_{\mathrm{in}, i}^{(s)}(k)+\gamma_{\mathrm{d}}\left(\tilde{\mathbf{w}}_{\mathrm{in}, i}^{(s)}(k)-\tilde{\mathbf{w}}_{\mathrm{out}, \mathcal{N}_{i}}^{(s)}(k)\right),
$$

where $\gamma_{\mathrm{d}}$ is a parameter that allows to tune the convergence of the iterative coordination process. Iterations stop when controllers fulfill the following local stopping condition at time instant $k$, e.g., for controller $i$ :

$$
\left\|\tilde{\lambda}_{\mathrm{in}, i}^{(s+1)}(k)-\tilde{\lambda}_{\mathrm{in}, i}^{(s)}(k)\right\|_{\infty} \leq \gamma_{\epsilon},
$$

where $\gamma_{\epsilon}$ serves as a numerical tolerance. Let $\tilde{\mathbf{V}}_{\text {inter }, i}(k)=$ $\left[\tilde{\mathbf{w}}_{\mathrm{in}, i}^{\mathrm{T}}(k) \tilde{\mathbf{w}}_{\mathrm{ou}, i}^{\mathrm{T}}(k)\right]^{\mathrm{T}}$. For the local cost $J_{\text {local }, i}$, the so called interconnecting cost (21) can be reformulated as:

$$
\begin{aligned}
& J_{\text {inter }, i}\left(\tilde{\mathbf{V}}_{\text {inter }, i}(k)\right) \\
& \quad=\tilde{\mathbf{V}}_{\text {inter }, i}^{T}(k) \mathbf{Q}_{\text {inter }, i} \tilde{\mathbf{V}}_{\text {inter }, i}(k)+\mathbf{f}_{\text {inter }, i}^{\mathrm{T}} \tilde{\mathbf{V}}_{\text {inter }, i}(k)+c_{\text {inter }, i}
\end{aligned}
$$

such that the augmented local optimization problem of controller $i$ is now:

$$
\min _{\tilde{\mathbf{V}}_{i}(k)} J_{i}\left(\tilde{\mathbf{V}}_{i}(k)\right)=\tilde{\mathbf{V}}_{i}^{T}(k) \mathbf{Q}_{i} \mathbf{V}_{i}(k)+\mathbf{f}_{i}^{T} \tilde{\mathbf{V}}_{i}(k)
$$

subject to

$$
\begin{aligned}
\mathbf{A}_{\text {in }, i} \tilde{\mathbf{V}}_{i}(k) & \leq \mathbf{b}_{\text {in }, i} \\
\mathbf{A}_{\text {eq }, i} \tilde{\mathbf{V}}_{i}(k) & =\mathbf{b}_{\text {eq }, i},
\end{aligned}
$$

where $\quad \tilde{\mathbf{V}}_{i}(k)=\left[\begin{array}{ll}\tilde{\mathbf{V}}_{\text {local }, i}^{\mathrm{T}}(k) & \tilde{\mathbf{V}}_{\text {inter }, i}^{\mathrm{T}}(k)\end{array}\right]^{\mathrm{T}}, \quad \mathbf{Q}_{i}=$ $\operatorname{diag}\left(\mathbf{Q}_{\text {local }, i}, \mathbf{Q}_{\text {inter }, i}\right)$ and $\mathbf{f}_{i}=\left[\mathbf{f}_{\text {local }, i}^{\mathrm{T}} \mathbf{f}_{\text {inter }, i}^{\mathrm{T}}\right]^{\mathrm{T}}$. The matrices in (26) are computed from $\mathbf{A}_{\text {in,local }, i}$ and $\mathbf{b}_{\text {in,local }, i}$ and include equalities (18)-(19). This is a standard QP problem that is solved by controller $i$.

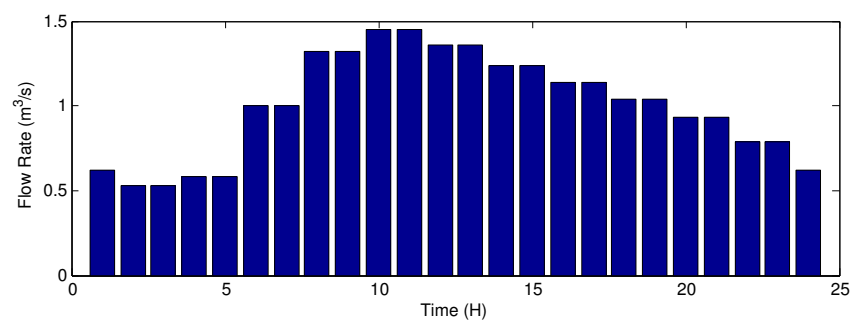

Fig. 2. A typical water demand profile.

It is worth mentioning that hard constraints (26) can be softened by introducing an additional positive slack variable $\epsilon$ in $\tilde{\mathbf{V}}_{i}(k)$ so that (26) becomes $\mathbf{A}_{\text {in }} \tilde{\mathbf{V}}_{i}(k) \leq \mathbf{b}_{\text {in }}+\epsilon \mathbf{1}$ where $\mathbf{1}$ is a unit column vector, and by adding an extra term $\rho \epsilon$ in the objective function $J_{i}$ of (25). The parameter $\rho$ allows to penalize the maximum amount of constraint violation.

\section{Simulation EXPERIMENT}

We next consider an experiment on the northern part water supply network of Bogotá (as indicated in Figure 1). This part of the network is modeled with 37 head and flowrate state variables, 7 control inputs (valves and pumping stations), and 18 disturbance inputs (water demand). The system is divided into three subnetworks indicated by letters A, B and C, the state dimensions of which are 10, 10, and 17 respectively. The interconnecting variables that interconnect the subnetworks consist of the head differences between the ends of interconnecting pipes. Each subnetwork is controlled by a local MPC controller that uses a discrete-time linearized prediction model. The control sampling is $1 \mathrm{~h}$, equaling the average time-constant of the system. The controllers use Matlab's quadprog function to perform the optimization required at each control time step.

The disturbance scenario considered in this experiment reflects typical water demand profiles, such as the one illustrated in Figure 2. The demand profile consists of flow rates specified for particular locations in the network at which consumers are connected over a time span of $24 \mathrm{~h}$.
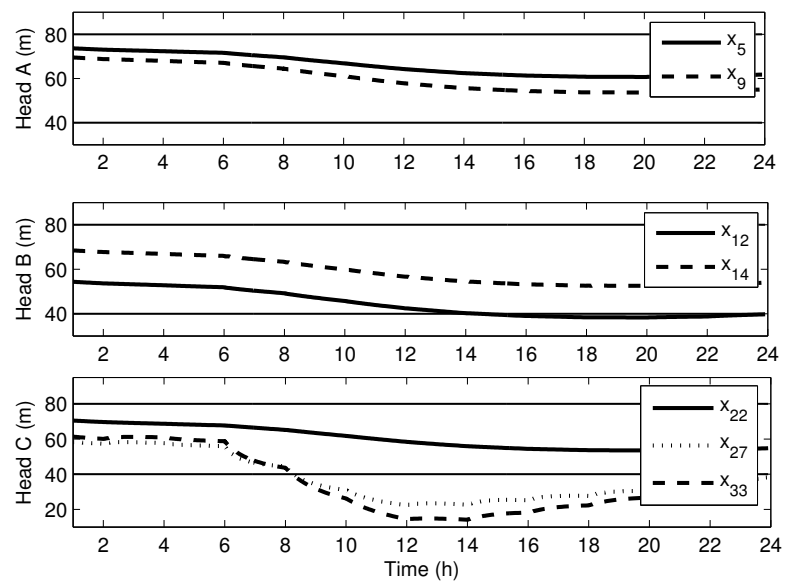

Fig. 3. Evolution of most important heads for each subnetwork over a full simulation without coordination. The horizontal lines indicate the allowed limits. 

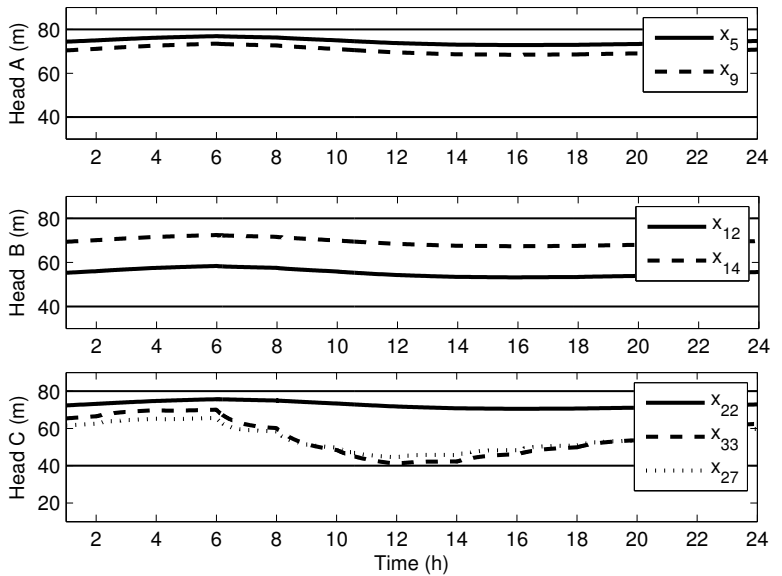

Fig. 4. Evolution of the most important heads for each subnetwork over a full simulation with coordination. The horizontal lines indicate the allowed limits.

The distributed control scheme presented in Section III has been applied to the three interconnected subnetworks. When facing a change in water demand, completely decentralized MPC controllers do not choose the best actions to apply to the network, as they do not take into account the actions of other controllers. This results in poor performance and economic losses, as heads and flow rates cannot be guaranteed to stay within acceptable limits as is seen in Figure 3.

However, when using the proposed distributed MPC approach to achieve coordination, the coordination between controllers allows to maintain the heads between the limits, while using the actuators optimally, as illustrated in Figure 4.

The results presented in Figure 4 have been obtained with the following choice of coordination parameters: $\gamma_{c}=10^{-1}$ in Eq. (21) and $\gamma_{d}=1$ in (22). The parameter $\gamma_{\epsilon}$ which appears in the stopping condition (23) is used to set a minimum level of coordination for the distributed scheme and is taken equal to $10^{-3}$.

\section{CONCLUSIONS AND FUTURE RESEARCH}

In this paper we have proposed the application of distributed model predictive control (MPC) for coordinating the actuators in large-scale water supply networks. We have introduced the characteristics of water supply networks, described how to model these systems, and discussed their control objectives. For control of such systems we have proposed the use of a distributed MPC scheme based on linearized models. A simulation study on a part of the water supply network of Bogotá, the capital of Colombia, shows the potential of the approach proposed.

Future research will further assess the performance of the proposed approach and implement the distributed MPC approach for the complete water network of Bogotá. Scalability issues, convergence properties, and robustness against uncertainty will then in particular be studied. Furthermore, the distributed MPC approach will be extended to nonlinear instead of linearized models.

\section{ACKNOWLEDGMENTS}

Research supported by the BSIK project "Next Generation Infrastructures (NGI)", the Delft Research Center Next Generation Infrastructures, and the European STREP project "Hierarchical and distributed model predictive control (HD-MPC)".

\section{REFERENCES}

[1] K. Vairavamoorthy, S. D. Gorantiwar, and A. Pathirana, "Managing urban water supplies in developing countries - Climate change and water scarcity scenarios," Physics and Chemistry of the Earth, vol. 33, no. 5, pp. 330-339, 2008.

[2] T. M. Walski, D. V. Chase, D. A. Savic, W. Grayman, S. Beckwith, and E. Koelle, Advanced Water Distribution Modeling and Management. Waterbury, Connecticut: Haestead Press, 2003.

[3] L. W. Mays, Hydraulic Design Handbook. New York, New York: McGraw-Hill Professional Publishing, 1999.

[4] J. M. Maciejowski, Predictive Control with Constraints. Harlow, UK: Prentice-Hall, 2002.

[5] K. Akouz, A. Benhammou, P. O. Malaterre, B. Dahbou, and G. Roux, "Predictive control applied to ASCE canal 2," in Proceedings of the 1998 IEEE International Conference on Systems, Man, and Cybernetics, San Diego, California, Oct. 1998, pp. 3920-3924.

[6] B. T. Wahlin and A. J. Clemmens, "Automatic downstream water-level feedback control of branching canal networks: Simulation results," Journal of Irrigation and Drainage Engineering, vol. 132, no. 3, pp. 208-219, May 2006.

[7] O. Begovich, V. M. Ruiz, G. Besançon, C. I. Aldana, and D. Georges, "Predictive control with constraints of a multi-pool irrigation canal prototype," Latin American Applied Research, vol. 37, no. 3, pp. 177185, Aug. 2007.

[8] T. Barjas Blanco, P. Willems, B. De Moor, and J. Berlamont, "Flood prevention of the Demer using model predictive control," in Proceedings of the 17th IFAC World Congress, Seoul, Korea, July 2008, pp. 3629-3634

[9] D. Georges, "Decentralized adaptive control for a water distribution system," in Proceedings of the 3rd IEEE Conference on Control Applications, Glasgow, UK, 1994, pp. 1411-1416.

[10] H. El Fawal, D. Georges, and G. Bornard, "Optimal control of complex irrigation systems via decomposition-coordination and the use of augmented Lagrangian," in Proceedings of the 1998 International Conference on Systems, Man, and Cybernetics, San Diego, California, 1998, pp. 3874-3879.

[11] S. Sawadogo, R. M. Faye, P. O. Malaterre, and F. Mora-Camino, "Decentralized predictive controller for delivery canals," in Proceedings of the 1998 IEEE International Conference on Systems, Man, and Cybernetics, San Diego, California, 1998, pp. 3380-3884.

[12] R. R. Negenborn, P. J. van Overloop, T. Keviczky, and B. De Schutter, "Distributed model predictive control for irrigation canals," Networks and Heterogeneous Media, vol. 4, no. 2, pp. 359-380, June 2009.

[13] I. Eker and T. Kara, "Operation and control of a water supply system," ISA Transactions, vol. 42, no. 3, pp. 461-473, July 2003.

[14] T. M. Walski, D. V. Chase, D. A. Savic, W. Grayman, S. Beckwith, and E. Koelle, Advanced Water Distribution Modeling and Management. Waterbury, Connecticut: Haestad Methods, 2003.

[15] R. R. Negenborn, B. De Schutter, and J. Hellendoorn, "Multi-agent model predictive control for transportation networks: Serial versus parallel schemes," Engineering Applications of Artificial Intelligence, vol. 21, no. 3, pp. 353-366, Apr. 2008. 